\title{
Effect of Granule Shape on the Giant Magnetoresistance for Ferromagnetic Metal-Metal Granular Films
}

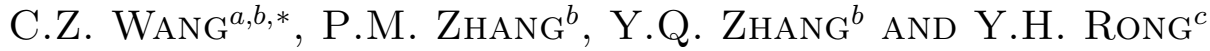 \\ ${ }^{a}$ Laboratory of Advanced Materials, Department of Materials Science and \\ Engineering, Tsinghua University, Beijing 100084, P.R. China \\ ${ }^{b}$ Department of Physics, Liaocheng University \\ Liaocheng 252059, Shandong Province, P.R. China \\ ${ }^{c}$ School of Materials Science and Engineering, Shanghai Jiao Tong University \\ Shanghai 200030, P.R. China
}

(Received August 20, 2007; revised version October 11, 2007)

\begin{abstract}
In order to investigate the effect of granule shape on the giant magnetoresistance, assuming that granular films consist of ellipsoidal ferromagnetic granules embedded in a nonmagnetic metal matrix and the ellipsoidal granules have different demagnetizing factors in three directions, we combined two-current model with the effective medium theory to investigate the effect of granule shape on the giant magnetoresistance. The results revealed that the giant magnetoresistance in granular films depended strongly on the granule shape and was between those for current perpendicular to the plane of the layers and current in the plane of the layers in magnetic multilayers.
\end{abstract}

PACS numbers: 72.15.Gd, 75.47.-m, 75.50.-y

\section{Introduction}

Since the discovery of giant magnetoresistance (GMR) in granular films in $1992[1,2]$, this phenomenon has been extensively investigated because it provided a new perspective in the information industry and enriched the mechanism of GMR [3-6]. The GMR in granular films originates primarily from the spin dependent scattering of conduction electrons at the interfaces between the ferromagnetic granules and the non-magnetic metallic matrix as well as within the ferromagnetic granules, which gives rise to the fact that GMR in granular films depends strongly on the granule size and granule shape. As soon as granule size is considered, the

*corresponding author; e-mail: wcz102@sjtu.org 
tendency is introduced that the GMR increases at first and then decreases with increasing granule size, reaching a peak value at a certain granule size [7-9] . However, it is so far conflicting about the effect of granule shape on the GMR. Sang et al. found that spherical shape granules are beneficial to the GMR [10]. However, Kataoka et al. indicated that granules with a pancake-like shape are advantageous to the GMR [11]. In order to clarify the above-mentioned contradiction, we combined the effective medium theory with the two-current model to investigate the effect of granule shape on the GMR in detail.

\section{Theoretical model}

We consider an ellipsoidal granule with conductivity $\sigma_{f}$ dispersed heterogeneously in a nonmetallic matrix with conductivity $\sigma_{n}$, as shown in Fig. 1. $a, b$, and $c$ are the lengths of the axes in the three directions of the ellipsoidal granule, respectively. In addition, assuming that the electric field $\left(\boldsymbol{E}_{0}\right)$ is parallel to $a$ axis and the applied magnetic field is parallel to the $c$ axis. This configuration resembles the case of transverse GMR. As well known, in most granular films the transverse GMR is the same as the longitudinal GMR except in some films containing ferromagnetic metal, such as $\mathrm{Ni}$, FeNi, CoNi $[12,13]$, therefore, we here consider the transverse GMR the same as the longitudinal GMR for convenience. Meanwhile, assuming that $L_{x}, L_{y}, L_{z}$ are the demagnetizing factors corresponding to the axis $a, b, c$ respectively. For simplicity, we only consider granules as rotational ellipsoids and let $b=c$ and the axial ratio $m=a / b$.

For granules with an axial ratio $m$ larger than one, $L_{x}$ is expressed as follows [14]:

$$
L_{x}=\frac{1}{1-m^{2}}+\frac{m}{\sqrt{\left(m^{2}-1\right)^{3}}} \ln \left(m+\sqrt{m^{2}-1}\right) .
$$

For granules with an axial ratio $m$ smaller than one, $L_{x}$ is expressed as follows:

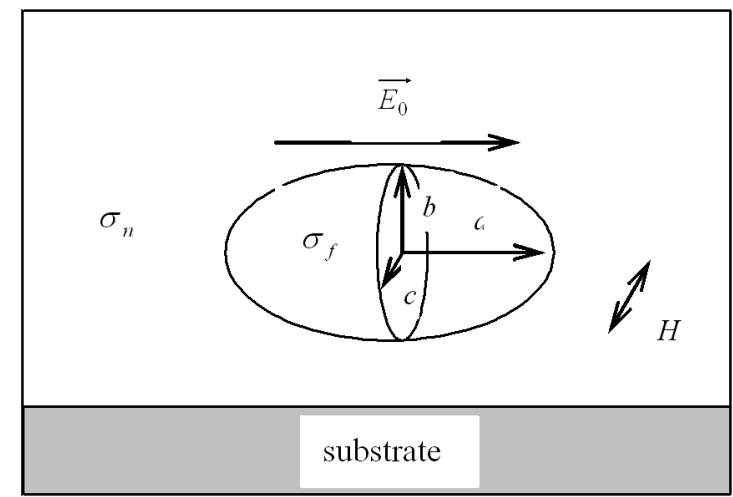

Fig. 1. Schematic diagram of an elliptical granule with conductivity $\sigma_{f}$ in a matrix with conductivity $\sigma_{n} . \boldsymbol{E}_{0}$ is the applied electric field. $\boldsymbol{H}$ is the applied magnetic field. 


$$
L_{x}=\frac{1}{1-m^{2}}-\frac{m}{\sqrt{\left(1-m^{2}\right)^{3}}} \arccos m .
$$

For granules with an axial ratio $m=1$ (spheres), $L_{x}=L_{y}=L_{z}=1 / 3$.

According to the two-channel conducting model, the scattering strength of electrons in spin-up and spin-down channels is different, giving rise to different conductivities in each channel in granular films. The scattering of electrons, whose spin is parallel to (say "spin-up"), the direction of the magnetic moment is very weak, leading to a high conductivity, while the scattering of electrons, whose spin is anti-parallel to (say "spin-down"), the direction of the magnetic moment is very strong, leading to a low conductivity. For a spin-up electron, the contribution to its resistance is low due to scattering events and then to its conductance is high, while for a spin-down electron, the contribution to its conductance is low. We use $\sigma^{+}$to represent high conductivity for the spin-up electrons and $\sigma^{-}$to represent low conductivity for the spin-down electrons. Here, the superscripts "+" and "-" stand for the direction of spin-up and spin-down electrons, respectively. In addition, it is known that the conductivity also depends on the number of conducting electrons per unit volume [15]. In the absence of an applied field, since the number of electrons in the spin-up and spin-down channel is equal, they have the same effect on the conductivity. However, in the existence of an applied field, the number of electrons in the two channels is uneven because electrons skip from the spin-down channel to the spin-up channel, resulting in the number of electrons in spin-up channel becoming larger than that in spin-down channel. Thus, inserting the coefficient " $a_{0}$ " and " $b_{0}$ " before the number of electrons in the existence of an applied field can describe this difference. It is obvious that $a_{0}>1$ (spin-up) and $b_{0}<1$ (spin-down) and $a_{0}+b_{0}=2$. Here, we take $a_{0}=1.4$ and $b_{0}=0.6$.

We consider the magnetic granule in the granular films as ellipsoids with a magnetic shell (the core conductivity $\sigma_{f}$ and the shell conductivity $\sigma_{m}$ ) embedded in an immiscible matrix (see Fig. 1). Liu and Li [16] considered that coated granules could be regarded as equivalent solid granules. Based on their method, the induced dipole moment of the coated ellipsoid, $P_{\alpha}$, is expressed by:

$$
P_{\alpha}=\frac{\lambda\left(2 \sigma_{m}^{\alpha}+\sigma_{n}\right)\left(\sigma_{f}^{\alpha}-\sigma_{m}^{\alpha}\right)+\left(\sigma_{m}^{\alpha}-\sigma_{n}\right)\left(\sigma_{f}^{\alpha}+2 \sigma_{m}^{\alpha}\right)}{2 \lambda\left(\sigma_{m}^{\alpha}+\sigma_{n}\right)\left(\sigma_{f}^{\alpha}-\sigma_{m}^{\alpha}\right)+\left(\sigma_{m}^{\alpha}+2 \sigma_{n}\right)\left(\sigma_{f}^{\alpha}+2 \sigma_{m}^{\alpha}\right)} E_{0},
$$

where $\alpha=+(-)$ denotes the spin direction of electrons, as mentioned above. $\lambda=a b^{2} /\left[(a+t)(b+t)^{2}\right]$, and $t$ is the thickness of the shell. In the quasi-static approach we can write $\sigma_{m}^{\alpha}$ as follows:

$$
\sigma_{m}^{\alpha}=\gamma_{\alpha} \sigma_{f}^{\alpha}
$$

where $\gamma_{\alpha}=\sigma_{m}^{k} / \sigma_{f}^{\alpha}$. Substituting Eq. (4) into Eq. (3) we can get another expression for induced dipole moment of the coated ellipsoid

$$
P_{\alpha}=\frac{\mu_{\alpha} \sigma_{f}^{\alpha}-\sigma_{n}}{\mu_{\alpha} \sigma_{f}^{\alpha}+2 \sigma_{n}} E_{0},
$$


where $\mu_{\alpha}$ is given by

$$
\mu_{\alpha}=\frac{\left(1-L_{x}\right) \gamma_{\alpha}+L_{x}+\left(1-L_{x}\right) \lambda\left(1-\gamma_{\alpha}\right)}{\left(1-L_{x}\right)+L_{x}-L_{x}\left(1-\gamma_{\alpha}\right)} .
$$

Taking the limit $t \rightarrow 0$, the interface resistance of granules $r_{\alpha}=t / \sigma_{m}^{\alpha}$ has a finite value, and $\mu_{\alpha}$ can be reduced to

$$
\mu_{\alpha}=\frac{a}{a+L_{x}(2 a / b+1) r_{\alpha} \sigma_{f}^{\alpha}} .
$$

In addition, the induced dipole moment of a simple solid ellipsoid can be given by

$$
P_{\alpha}=\frac{\sigma_{a}^{\alpha}-\sigma_{n}}{\sigma_{a}^{\alpha}+2 \sigma_{n}} E_{0} .
$$

Comparing the induced dipole moment of the coated ellipsoid, Eq. (5), with that of an equivalent solid ellipsoid, Eq. (8), we found that the conductivity of the equivalent solid ellipsoids is expressed as follows:

$$
\sigma_{a}^{\alpha}=\mu_{\alpha}=\mu_{\alpha} \sigma_{f}^{\alpha} .
$$

According to the effective medium theory [17], the average field $(\boldsymbol{E})$ and current density $(\boldsymbol{J})$ are given by

$$
\begin{aligned}
& \left\langle\boldsymbol{E}_{\uparrow(\downarrow)}\right\rangle=f \boldsymbol{E}_{1, \alpha}+(1-f) \boldsymbol{E}_{0} \\
& =f \frac{\sigma_{n}}{\sigma_{n}+\left(\sigma_{a}^{\alpha}-\sigma_{n}\right) L_{x}}+(1-f) \sigma_{n} \boldsymbol{E}_{0}, \\
& \left\langle\boldsymbol{J}_{\uparrow(\downarrow)}\right\rangle=f \sigma_{a}^{\alpha} \boldsymbol{E}_{1, \alpha}+(1-f) \sigma_{n} \boldsymbol{E}_{0},
\end{aligned}
$$

where $\uparrow(\downarrow)$ represents the magnetization direction of ferromagnetic granules on the basis of assumption that all ferromagnetic granules have only two magnetization directions [18], and $f$ represents the volume fraction of ferromagnetic granules, subscript "1" stands for the ferromagnetic granules. Therefore, the effective conductivity in the Maxwell-Garnett approximation of a coated ellipsoidal granule is

$$
\sigma^{\mathrm{eff}}=\sigma_{n} \frac{L_{x} \sigma_{a}^{\alpha}+\left(1-L_{x}\right) \sigma_{n}+f\left(1-L_{x}\right)\left(\sigma_{a}^{\alpha}-\sigma_{n}\right)}{L_{x} \sigma_{a}^{\alpha}+\left(1-L_{x}\right) \sigma_{n}+f L_{x}\left(\sigma_{a}^{\alpha}-\sigma_{n}\right)} .
$$

Here, we define $G M R$ as

$$
G M R=\frac{\sigma_{\mathrm{M}}-\sigma_{\mathrm{D}}}{\sigma_{D}},
$$

where $\sigma_{\mathrm{M}}$ and $\sigma_{\mathrm{D}}$ stand for the conductivity of the granular films in saturated applied field $H_{\mathrm{s}}$ and in a completely demagnetized state, respectively. For the completely magnetized state, the moments of all granules have the same direction parallel to the applied field, and the conductivity of the granular films can be written as follows:

$$
\sigma_{\mathrm{M}}=\frac{a_{0} f Q^{+} \sigma_{a}^{+}+(1-f) \sigma_{n}}{f Q^{+}+1-f}+\frac{b_{0} f Q^{-} \sigma_{a}^{-}+(1-f) \sigma_{n}}{f Q_{-}+1-f},
$$

where 


$$
\begin{aligned}
Q^{+} & =\frac{\sigma_{n}}{\sigma_{n}+\left(a_{0} \sigma_{a}^{+}-\sigma_{n}\right) L_{x}}, \\
Q^{-} & =\frac{\sigma_{n}}{\sigma_{n}+\left(b_{0} \sigma_{a}^{-}-\sigma_{n}\right) L_{x}} .
\end{aligned}
$$

In a completely demagnetized state, both the number and the scattering strength of electrons in the spin-up and spin-down channels are identical, indicating that $a_{0}=b_{0}=1$. Therefore, the effective conductivity can be written as follows:

$$
\sigma_{\mathrm{D}}=\frac{f\left(a_{0} Q^{+} \sigma_{a}^{+}+b_{0} Q^{-} \sigma_{a}^{-}\right)+2(1-f) \sigma_{n}}{0.5 f\left(Q^{+}+Q^{-}\right)+1-f} .
$$

\section{Results and discussion}

Using the above-mentioned theory, we investigate the GMR of films in the case where the spin diffusion length is much longer than both the mean free path and the granule size so that the spin-flip effect can be neglected and the two-current model is applicable.

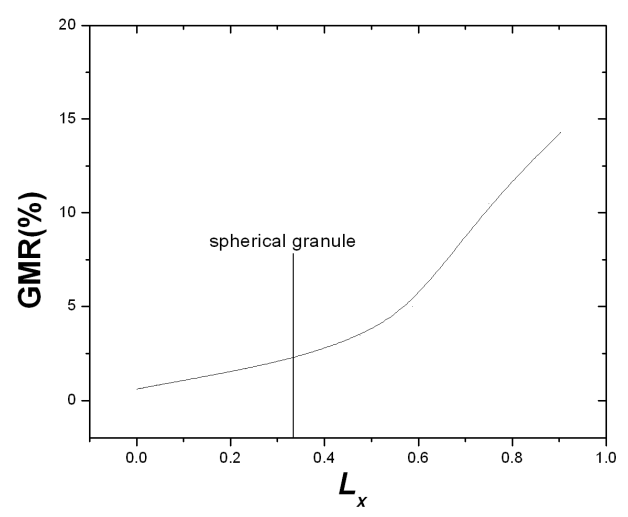

Fig. 2. The dependence of $G M R$ on the demagnetizing factor, $f=0.2, \sigma_{n}=4.2$ $b=10 r_{-} \sigma_{f}^{-}, \sigma_{f}^{+} / \sigma_{f}^{-}=20, \sigma_{m}^{+} / \sigma_{m}^{-}=20$.

Figure 2 shows the dependence of $G M R$ on the demagnetizing factor, i.e., the dependence of $G M R$ on the granule shape. It is clear that the $G M R$ increases monotonically with increasing demagnetizing factor. Here we discuss two extreme cases: one is that $a \rightarrow 0$ and $c \rightarrow \infty$, and the other one is when $a \rightarrow \infty$ and $c \rightarrow 0$. When $a \rightarrow 0$ and $c \rightarrow \infty, L_{x} \rightarrow 1$ and the GMR is large. In this case, the granule has a shape with a very large pancake perpendicular to the electric field, which resembles the case of current perpendicular to the plane (CPP) of the layers in a multilayer. However, when $a \rightarrow \infty$ and $c \rightarrow 0, L_{x} \rightarrow 0$ and the $G M R$ is small. In this case, all the granules form a shape with a very large pancake parallel to the electric field, which resembles the case of current in the plane (CIP) of layers in a multilayer. In practice, the shape of granules in films is ellipsoid, that is to say, the demagnetizing factor of granules is between 0 and 1 , indicating that the 


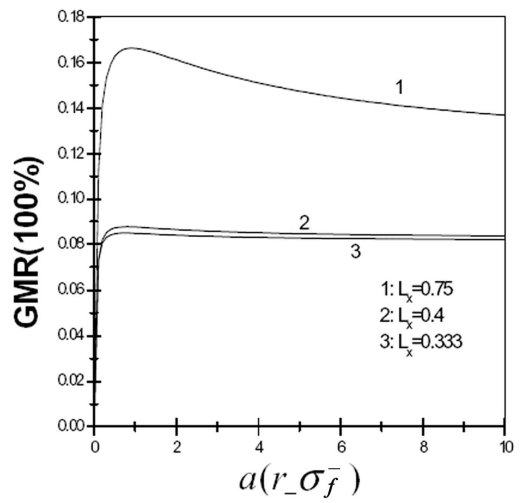

Fig. 3. The dependence of $G M R$ on the size of granule with various demagnetizing factors, $f=0.2, \sigma_{n}=4.2, \sigma_{f}^{+} / \sigma_{f}^{-}=20, \sigma_{m}^{+} / \sigma_{m}^{-}=20$.

GMR of granular films is between that of CPP case and that of CIP case in a multilayer, which is in agreement with the results of $\mathrm{Gu}$ et al. [9]. On the other hand, these results show that granules with a pancake-like shape are advantageous to the GMR, which is identical to the results of Kataoka et al. [11]. In addition, it should be pointed out that the above formula is inapplicable in these two extreme cases with $L_{x} \rightarrow 1$ or $L_{x} \rightarrow 0$ in GMR.

Figure 3 shows the dependence of $G M R$ on the size of the granule with various demagnetizing factors. It is clear that the $G M R$ first increases and then decreases with increasing granule size, which is in agreement with the experiment of Wang et al. [7], and a maximum value occurs at a certain granule size with any demagnetizing factor. It is known from the physical point of view that on the one

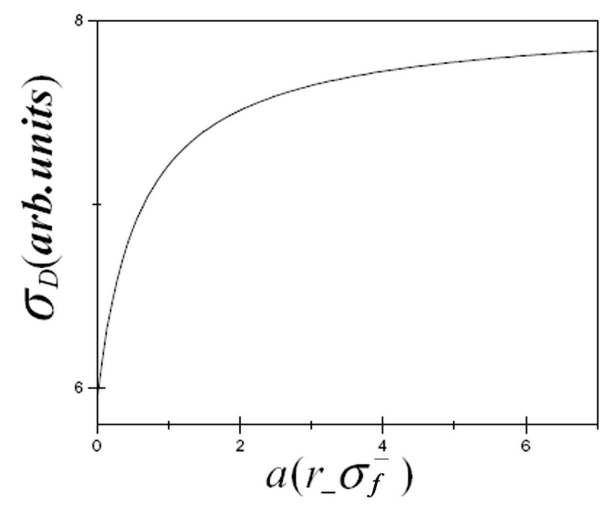

Fig. 4. The dependence of the conductivity $\sigma_{\mathrm{D}}$ on the granule size, $f=0.2, \sigma_{n}=4.2$, $L_{x}=0.4, \sigma_{f}^{+} / \sigma_{f}^{-}=20, \sigma_{m}^{+} / \sigma_{m}^{-}=20$. 
hand, the surface-to-volume ratio of the granules increases with decreasing granule size, giving rise to the enhancement of the spin-dependent interface scattering and the GMR effect; on the other hand, when the granule size decreases to below the mean free path of electrons, the currents pass by granules more easily, giving rise to the reduction of spin-dependent interface scattering and the GMR effect. The competition between these two factors leads to a maximum value of the GMR at a certain granule size.

Figure 4 shows the dependence of the conductivity $\cdot \sigma_{D}$ on the granule size. It can be seen that the conductivity increases with increasing granule size, namely, the resistivity decreases with increasing granule size, which is in agreement with the experiment of Xiao et al. [19] that the resistivity decreases with increasing annealing temperature since the granular size increases monotonically with the elevation of annealing temperature.

\section{Conclusions}

In summary, we combined the two-current model with effective medium theory to investigate the GMR of granular films. The results indicate that the GMR of granular films is between the CIP and CPP cases in a multilayer and granules with a pancake-like shape are advantageous to the GMR. In addition, there is an optimum granule size for the GMR and the conductivity of films in the absence of the applied field increases monotonically with increasing granule size.

\section{Acknowledgments}

This work is financially supported by the Nature Science Foundation of Shandong Province under Grant No. 2005zx11 and No.Y2006A02, and the National Nature Science Foundation of China under Grant No. 50071033.

\section{References}

[1] A.E. Berkowitz, J.R. Mitchell, M.J. Corey, A.P. Young, S. Zhang, F.E. Spada, F.T. Parker, A. Hutten, G. Thomas, Phys. Rev. Lett. 68, 3745 (1992).

[2] J.Q. Xiao, J.S. Jiang, C.L. Chien, Phys. Rev. Lett. 68, 3749 (1992).

[3] N. Peleg, S. Shtrikman, G. Gorodetsky, I. Felner, J. Magn. Magn. Mater. 191, 349 (1999).

[4] Y. Ju, Xu Chen, Z.Y. Li, J. Magn. Magn. Mater. 233, 267 (2001).

[5] A. Milner, I.Y. Korenblit, A. Gerber, Phys. Rev. B 60, 14821 (1999).

[6] G.J. Strijkers, H.J.M. Swagten, B. Rulkens, R.H.J.N. Bitter, W.J.M. De Jonge, P.J.H. Bloemen, K.M. Schep, J. Appl. Phys. 84, 2749 (1998).

[7] W.D. Wang, F.W. Zhu, J. Weng, J.M. Xiao, W.Y. Lai, Appl. Phys. Lett. 72, 1118 (1998).

[8] S. Zhang, P.M. Levy, J. Appl. Phys. 69, 4786 (1991).

[9] R.Y. Gu, L. Sheng, D.Y. Xing, Z.D. Wang, J.M. Dong, Phys. Rev. B 53, 11685 (1996). 
[10] H. Sang, Z.S. Jiang, G. Guo, J.T. Ji, S.Y. Zhang, Y.W. Du, J. Magn. Magn. Mater. 140/144, 589 (1995).

[11] N. Kataoka, H. Takeda, J. Echigoya, K. Fukamichi, E. Aoyagi, Y. Shimada, H. Okuda, K. Osamura, M. Furusaka, T. Goto, J. Magn. Magn. Mater. 140/144, 621 (1995)

[12] W. Thomson, Proc. R. Soc. 8, 546(1857).

[13] C.Z. Wang, Y.H. Rong, T.Y. Hsu, Materials Science Poland 24, 351 (2006).

[14] W.D. Zhong, Ferromagnetism, Science Press, Beijing 1987, p. 51 (in Chinese).

[15] Y. Ju, Z.Y. Li, Phys. Lett. A 277, 169 (2000).

[16] X. Liu, Z.Y. Li, Phys. Lett. A 223, 475 (1996).

[17] T.C. Choy, Effective Medium Theory, Clarendon Press, Oxford 1999, p. 1.

[18] R. Yang, W.J. Song, Phys. Lett. A 244, 139 (1998).

[19] J.Q. Xiao, J.S. Jiang, C.L. Chien, IEEE Trans. Magn. 29, 2688 (1993). 